\title{
¿NADIE SABE LO QUE PUEDE UN CUERPO? HISTORIA DE LA INVERSIÓN DE UN ERROR
}

\author{
NO ONE KNOWS WHAT A BODY CAN DO? \\ HISTORY OF THE INVERSION OF AN ERROR
}

\author{
Claudia Aguilar ${ }^{1}$ \\ Conicet
}

Recibido: 06.04.2020 - Aceptado: 25.10.20

\begin{abstract}
RESUMEN
La frase "nadie sabe lo que puede un cuerpo" se la retoma generalmente para plantear la indeterminación de los cuerpos o el poder ilimitado de ellos. Sin embargo, algo que no se tiene en cuenta es que uno de los puntos centrales de la filosofía spinoziana es la doble atribución; por lo que mente y cuerpo son una sola y misma cosa vista desde distintos atributos de la única sustancia. Para explicar esta frase, que nunca aparece formulada de esa manera en las obras de Spinoza, considero que es crucial la noción de individuo. Mi hipótesis es que dicha afirmación es inadecuada si partimos de la doble atribución y si consideramos las caracterizaciones del individuo. Mis objetivos son dos: (1) desarrollar las caracterizaciones del individuo como cuerpo complejo y conatus para abordar cómo estas caracterizaciones responden a la pregunta por lo que puede un cuerpo y (2) analizar el escolio que dio lugar a la frase del título, frase que invierte erróneamente un viejo error.
\end{abstract}

Palabras clave: Spinoza; Individuo; Relación; Conatus; Cuerpo.

\section{ABSTRACT}

"No one knows what a body can do" is one of the most invoked phrases about Spinoza; generally to talk about the indetermination of bodies or the unlimited power of them. However, that does not take into account the double attribution, one of the central points of Spinoza's philosophy; so mind and body are one and the same thing seen from different attributes of the unique substance. To explain this phrase, which never appears like that in Spinoza's works, I consider the notion of individual is crucial. My hypothesis is that this statement is inadequate if we consider the double attribution and the characterizations of the individual. I have two goals: (1) to develop the characterizations of the individual as a complex body and conatus; in order to to address how these characterizations answer the question about what a body can do; and (2) to analyze the scholium that gave rise to the phrase in the title, phrase that erroneously reverses an old error.

Keywords: Spinoza; Individual; Relationship; Conatus; Body.

${ }^{1}$ claudiamaaguilar@gmail.com 


\section{INTRODUCCIÓN}

"Nadie sabe lo que puede un cuerpo" ha sido una de las frases más utilizadas a la hora de referirse a la filosofía de Spinoza. Se afirma a partir de ella la imposibilidad de conocimiento sobre los cuerpos, su potencia ilimitada o la indeterminación de ellos. Sin embargo, más allá de que esta frase no se encuentre en la letra de Spinoza, lo anterior implica perder de vista la doble atribución de dicha filosofía. Doble atribución que establece que mente y cuerpo son una sola y misma cosa vista desde distintos atributos de la única sustancia. No hay ni libre albedrío donde la mente manda y el cuerpo obedece; pero tampoco indeterminación del cuerpo.

Lo que considero la clave para entender que esa frase es inadecuada es el análisis de la noción de individuo. El individuo es definido por Spinoza en la digresión física de su Ética como un cuerpo complejo (E II, prop. 13, def.). ${ }^{2}$ Allí este es caracterizado como la relación de movimiento y reposo que mantienen las partes que lo conforman. Esta caracterización del individuo como relación ha tenido una crítica muy fuerte: la de ser un criterio insuficiente para determinar la individualidad. ${ }^{3}$ Mi respuesta a dicha crítica, cómo desarrollaré en el apartado siguiente, es que esta definición se complementa con la caracterización del individuo como conatus. ${ }^{4} \mathrm{La}$ hipótesis que quiero defender es que la afirmación "nadie sabe lo que puede un cuerpo" es inadecuada si partimos de la doble atribución y si consideramos las caracterizaciones del individuo. Para demostrar que es posible saber lo que puede un cuerpo comenzaré por el análisis de la noción de individuo como relación de movimiento y reposo y como conatus. Desarrollaré brevemente los afectos, en tanto que variaciones de la potencia.

2 Cito la Ethica ordine geometrico demonstrata, como es habitual, utilizando la abreviatura E, seguida de la parte con números romanos y las siguientes abreviaturas y números arábigos para indicar las diferentes secciones: def. (definición), ax. (axioma), prop. (proposición), lem. (lema), post. (postulado), esc. (escolio), cor. (corolario), ap. (apéndice), pref. (prefacio), def. gral. de los af. (definición general de los afectos). Utilizo la traducción castellana de Atilano Domínguez consignada en la bibliografía.

3 Como ejemplo de esta línea interpretativa podemos nombrar a Martin Lin, quien sostiene: "Spinoza's account of ratio thus need not have the wildly implausible consequences that some commentators have thought it has. We might still wonder if Spinoza's account of ratio is sufficiently robust to do the work that Spinoza needed it to do. To say that what makes a thing the same thing over time and through change is sameness of ratio is, it might seem, extremely unhelpful." (Lin 2005, 252). 2019.

4 Para una tercera caracterización de individuo como cosa singular véase Aguilar 
En segundo lugar, analizaré en detalle el escolio que dio lugar a la frase del título, para concluir que lo que puede un cuerpo consiste en lo que aparece en la digresión física junto con la potencia con la que se esfuerza por perseverar en el ser. El eje que atraviesa la argumentación es la doble atribución spinoziana, que considerada junto con las caracterizaciones del individuo como cuerpo complejo y conatus, revela la frase "nadie sabe lo que puede un cuerpo" como inadecuada.

\section{INDIVIDUO: CUERPO COMPLEJO Y CONATUS}

El primer paso para comprender lo que puede un cuerpo es dar un rodeo por la noción de individuo, ya que este es definido como un cuerpo complejo. La definición del individuo como cuerpo complejo aparece en la segunda parte de la Ética. Allí el individuo es definido según la relación que mantienen sus partes:

Cuando algunos cuerpos de la misma o distinta magnitud son forzados por otros a que choquen entre sí o, si se mueven con el mismo o con distintos grados de rapidez, a que se comuniquen unos a otros sus movimientos en cierta proporción; diremos que dichos cuerpos están unidos entre sí y que todos a la vez forman un solo cuerpo o individuo que se distingue de los demás por esta unión de cuerpos. (E II, prop. 13, def.)

Para conformar un individuo tiene que haber cuerpos que se comunican entre sí el movimiento y el reposo según una cierta relación. No basta con comunicar los movimientos; conjuntamente, es necesaria la existencia de otros cuerpos presionando. Ambas cosas deben suceder. La individualidad, entonces, estará dada por la mantención de la relación del movimiento y el reposo de las partes junto con la continuidad de los cuerpos externos que presionan ${ }^{5}$ y con los que se relaciona. Esta definición se encuentra en la llamada digresión física, sección posterior a la proposición 13 de la segunda parte de la obra y en la que, además de la definición citada, encontramos una serie de axiomas, lemas y postulados. Sin entrar en detalle en la digresión, lo que me interesa resaltar es que allí Spinoza realiza un estudio de los cuerpos en términos estrictamente corporales, estableciendo sus propiedades y no remitiéndose al atributo pensamiento. Según la digresión física, un cuerpo puede estar en reposo o moverse (E II, prop. 13, ax. 1) más rápida o más lentamente (E II, prop. 13, ax. 2). Cada cuerpo puede determinar o ser determinado por otro cuerpo al movimiento o al reposo ( $\mathrm{E}$ II, prop. 13, lem. 3) y sus partes pueden variar de múltiples maneras siendo

\footnotetext{
${ }^{5}$ Esto es lo que Gueroult llama "presión de ambiente”. (Cf. Gueroult 1974, 166).
} 
él el mismo (E II, prop. 13, lem. 4, 5, 6 y 7). Todo esto es, en parte, lo que puede un cuerpo.

Sin perder de vista la doble atribución spinoziana, la definición de individuo, al igual que el resto de la digresión, debe ser considerada como el correlato en la extensión de la relación entre ideas que se da en el atributo pensamiento. ${ }^{6}$ La definición de individuo de la digresión física tiene su correlato correspondiente en el atributo pensamiento. Así, por ejemplo, la segunda proposición una vez concluida la digresión física sostiene: "la idea que constituye el ser formal del alma humana no es simple, sino compuesta de muchísimas ideas" (E II, prop. 15). En la demostración es clara la correlación entre los dos atributos. Allí se sostiene que el cuerpo humano se compone de muchísimos individuos muy compuestos. Y, al ser la idea del cuerpo la que constituye el ser formal de la mente humana, hay en la sustancia una idea de cada individuo componente de ese cuerpo. ${ }^{7}$ Por lo cual, la idea del cuerpo humano está compuesta de muchas ideas de sus partes componentes (E II, prop. 15, dem). Queda así demostrada la complejidad de la mente humana.

Ahora bien, la definición de individuo de la segunda parte de la Ética es la más general y es insuficiente a la hora de brindar un criterio intrínseco del individuo; dado el rol preponderante que tienen los cuerpos exteriores en su conformación. Asimismo, para hablar "del origen y naturaleza de los afectos", como el nombre de la tercera parte lo indica, la definición analizada no alcanza. Dicho de otra manera: la definición física es insuficiente para dar cuenta de la esencia de los individuos y de las variaciones de la potencia de cada uno de ellos. Debemos abordar, entonces, el segundo aspecto de la individualidad: el individuo como conatus. Este segundo aspecto se aboca a la esencia de los individuos y nos ayudará a responder a la pregunta por lo que puede un cuerpo.

${ }^{6} \mathrm{Al}$ respecto de la correlación entre ambos atributos sostiene Lin: "This isomorphism allows us to infer that for every complex individual body, i.e., every collection of bodies that realizes a ratio of motion and rest, there is a complex individual idea (i.e., a mind) the parts of which (themselves ideas) relate to one another according to a ratio or pattern isomorphic to the one realized by the correlative complex body. Whereas an individual body maintains a ratio of motion and rest, an individual mind maintains a psychological ratio, perhaps a pattern of inference and association". (Lin 2005, 254). Es decir, dada la complejidad del cuerpo, debe inferirse la complejidad de la mente humana. En un sentido similar Peterman sostiene: "At E2p15, Spinoza leverages E27 to show that the human mind is composite, which suggests that he thinks the human mind has the same relationship to its parts as the human body does". (Peterman 2007, 117).

${ }^{7}$ Utilizo el término "mente" porque considero que es el que más se adecúa al utilizado por Spinoza: "mens". Sin embargo, en las citas textuales dicho término aparecerá como "alma” ya que así figura en la traducción. 


\section{Spinoza sostiene:}

Cada cosa, en cuanto está en ella, se esfuerza por perseverar en su ser

Demostración: En efecto, las cosas singulares son modos en los que se expresan de una cierta y determinada manera los atributos de Dios (por $1 / 25 \mathrm{c}$ ); es decir (por 1/34), son cosas que expresan de cierta y determinada manera la potencia de Dios, por la que Dios es y actúa. (E III, prop. 6)

Cada cosa se esfuerza por perseverar en su ser cuanto esté a su alcance, dado que cada cosa es un modo que expresa de cierta y determinada manera la potencia de la sustancia (E III, prop. 6, dem.). Más aún, este esfuerzo, o conatus, que realiza cada cosa por perseverar en su ser es la esencia actual de la cosa misma (E III, prop. 7). Una cosa solo puede ser destruida por una causa exterior, es decir, ninguna cosa puede ser causa de su propia destrucción (E III, prop. 4); ya que la definición de una cosa solo puede afirmar y no negar la esencia de esa cosa (E III, prop. 4, dem.). De este modo, no pueden darse cosas contrarias en un mismo individuo, en la medida en que una de ellas pueda destruir a la otra (E III, prop. 5). Y esto es de tal modo ya que no puede darse en el mismo individuo algo que tenga la capacidad de destruirlo (E III, prop. 5, dem.). La causa de la destrucción de una cosa siempre debe ser externa y por eso no puede haber cosas que se destruyan mutuamente en un mismo individuo. Pero lo que hace que cada cosa se esfuerce cuanto está a su alcance por perseverar en su ser es su relación con la sustancia, en tanto que es una expresión de esta.

En la demostración de la proposición 7 de E III, Spinoza presenta una equiparación que dará lugar a varios malentendidos entre sus lectorxs. Allí sostiene: "la potencia de cualquier cosa o el conato", y, también, "la potencia o el conato con el que se esfuerza por perseverar en su ser" (E III, prop. 7, dem.). Spinoza en esta demostración analoga explícitamente conatus y potencia. Sin embargo, esta equiparación no resulta del todo exacta. Una lectura precisa de los términos permite ver que el conatus es el esfuerzo con que cada cosa persevera en el ser y que este esfuerzo tiene un grado de potencia. ${ }^{8}$ Aunque esta diferenciación sea sutil, nos permitirá evitar

8 Por ejemplo, se ocasiona este malentendido cuando Deleuze sostiene: "En latín «esforzarse» se dice conor. El esfuerzo o la tendencia, el conatus. He aquí que la noción de límite está definida en función de un esfuerzo. Y la potencia es la tendencia misma o el esfuerzo en tanto que tiende hacia un límite". (Deleuze 2003, 373).

Otro ejemplo es Caroline Williams, quien sostiene: "The essence of a thing undergoes mutation and variation; reciprocity of relations enable or produce a transformation of essence and power (E2p10s; Deleuze 1988: 64). [...] Indeed, perhaps the conatus is this open series of power relations at the heart of every mode of existence: the power (of all things) to persist (and to desist, or resist); a configuration of forces that are internal and not prior to the process of individuation itself (for further discussion, see Williams 2017)". (Williams $2019,123)$. Sin embargo, no debemos perder de vista que el esfuerzo no varía, varía la 
confusiones en el presente trabajo; ya que el conatus mantiene mayor independencia de los cuerpos exteriores que el grado con el que se ejerce su potencia; dado que la potencia varía según las afecciones. La caracterización del individuo en tanto que conatus, esencia actual, es en tanto que esfuerzo por el que cada cosa, ya sola o con otras, intenta obrar algo. El conatus, entonces, es el esfuerzo por perseverar en el ser contra esas causas exteriores que podrían destruirlo. El conatus de un individuo es el esfuerzo de este cuya potencia tiene múltiples variaciones según los distintos afectos, es decir, las variaciones del cuerpo por las cuales aumenta o disminuye su potencia de actuar (E III, def.3).

Antes de pasar al análisis de las variaciones de la potencia, de los afectos activos y los afectos pasivos, vale la pregunta ¿qué particularidad suma esta caracterización del individuo como conatus a la ya mencionada caracterización como relación? Sostengo, en primer lugar, que hay una diferencia. En la caracterización de individuo como relación tenían un papel preponderante en la conformación del individuo los cuerpos exteriores que presionaban (E II, prop. 13, def.). En el segundo aspecto del individuo, el conatus, el acento está puesto en el propio esfuerzo intrínseco del individuo en tanto que expresión de la potencia de la sustancia. Si bien los cuerpos exteriores hacen que aumente o disminuya la potencia de ese individuo, o sea, la potencia con la que se esfuerza; ese esfuerzo, en tanto que esencia actual, es lo propio de ese individuo. He aquí la importancia de la distinción entre potencia y esfuerzo: el esfuerzo es expresión de la potencia de la sustancia, la potencia de los individuos finitos, en cambio, varía según las afecciones.

La esencia actual revela lo intrínseco del individuo, de ese cuerpo y esa mente complejos. En este sentido, considero que el segundo aspecto de la individualidad permite sortear ciertas críticas que la teoría de la individualidad spinoziana ha despertado, por ejemplo, la de Leibniz. La crítica leibniziana a Spinoza se enfoca en la noción de individuo. Según el filósofo alemán, en la filosofía de Spinoza la conservación de un cuerpo o un individuo no sucede en virtud de un esfuerzo intrínseco de este; al contrario, las mantención de los individuos sería gracias a que los cuerpos componentes sigan comunicándose sus movimiento. En esta crítica leibniziana Spinoza incurriría en la misma carencia que Leibniz le reprocha a Descartes: no contar con una caracterización intrínseca de los individuos (Leibniz 1923, 1715). ${ }^{9}$

potencia que tiene ese esfuerzo. Como se puede observar, no es posible hacer esta distinción si equiparamos conatus y potencia.

${ }^{9} \mathrm{Al}$ respecto, véase Jabase 2020. 
En un punto coincidiría con la crítica de Leibniz, la definición de individuo de la digresión física es necesaria pero no suficiente a la hora de caracterizar intrínsecamente la noción de individuo. La relación entre las partes, el movimiento y el reposo del lema 1, no llega a proveer un criterio inherente de la individualidad. Ahora bien, en realidad la crítica no es acertada porque el individuo no es caracterizado solamente en la digresión física. ${ }^{10} \mathrm{Y}$ a diferencia de la primera caracterización (de individuo como relación), en el conatus no figuran los cuerpos externos; y, de este modo, es una caracterización más intrínseca que la primera. ${ }^{11}$

Vemos hasta aquí que lo que puede un cuerpo (un cuerpo complejo, que es un individuo) comprende tanto el movimiento y el reposo como la potencia del esfuerzo por perseverar en el ser. Esfuerzo que es intrínseco en tanto expresión de la potencia de la sustancia, sin un rol preponderante de los cuerpos exteriores.

Ya hacia el final de la segunda parte de la Ética, aparece implícitamente lo que luego será definido como conatus. Spinoza establece que cada idea de un cuerpo, o de una cosa singular, implica la esencia eterna e infinita de la sustancia de manera necesaria (E II prop. 45). En el escolio afirma que lo que luego será definido como conatus se sigue de Dios más allá de la determinación intramodal, más allá de la determinación por otro modo finito; ya que si bien cada una es determinada por otra cosa singular a existir

10 Concuerdo aquí con Leila Jabase, quien afirma: "Ahora bien, es preciso decir, que las acusaciones que Leibniz realiza no se corresponden completamente con la concepción original de Spinoza, quien define una cosa individual justamente por su conatus o esencia actual y no solo por la conservación de la relación de reposo y movimiento. Tal como muchos estudiosos de su obra han remarcado, podría decirse que subyace a la crítica leibniziana una comprensión de Spinoza no del todo ajustada a su sistema, y que en cierta medida deforma el pensamiento del autor". (Jabase 2020).

11 Deleuze también distingue el conatus de la relación de movimiento y reposo a partir de considerar el última algo extrínseco. Así lo sostiene: "Una esencia de modo se expresa eternamente en una relación, pero no debemos confundir la esencia y la relación en la que se expresa. Una esencia de modo no es causa de la existencia del modo mismo: esta proposición retoma, en términos spinozistas, un viejo principio según el que la existencia de un ser finito no deriva de su esencia. Pero, ¿cuál es el nuevo sentido de este principio en las perspectivas de Spinoza? Significa lo siguiente: por mucho que una esencia de modo se exprese en una relación característica, no es ella la que determina una infinidad de partes extensivas a entrar bajo esa relación. (No es la naturaleza simple la que establece por ella misma su dominación, ni la que obliga ella misma a partes a ajustarse conformemente a la relación en la que se expresa.). Pues las partes extensivas se determinan unas a otras, desde el exterior y al infinito; no tienen otra determinación que extrínseca. Un modo pasa a la existencia, no en virtud de su esencia, sino en virtud de leyes puramente mecánicas que determinan una infinidad de partes extensivas cualesquiera a entrar bajo tal relación precisa, en la que su esencia se expresa. Un modo deja de existir desde que sus partes son determinadas a entrar bajo otra relación, correspondiente a otra esencia. Los modos pasan a la existencia, y dejan de existir, en virtud de leyes exteriores a sus esencias". (Deleuze 1999, 201-202). 
de cierta manera, lo anterior no quita que la fuerza en cuya virtud cada una de ellas persevera en la existencia se siga de la eterna necesidad de la naturaleza de la sustancia (E II, prop. 45, esc.). Acto seguido, Spinoza remite al corolario de la proposición 24 de la primera parte, donde afirma que la sustancia no es sólo causa del comienzo de la existencia de las cosas sino que es causa de que perseveren en la existencia (E I, prop. 24, corol.). A partir de lo anterior es que podemos sostener que el conatus - aunque no su grado de potencia- mantiene cierta independencia de la determinación intramodal (si bien la relación intramodal es lo que puede sacar a una cosa de la existencia); siendo así el conatus un aspecto más intrínseco del individuo que la relación de movimiento y reposo, relación en la que había un gran protagonismo de los cuerpos exteriores ${ }^{12}$ para su conformación. En este respecto, me distancio de la interpretación de Justin Steinberg quien, acentuando que el individuo es meramente la relación de movimiento y reposo, considera que la demanda de algo intrínseco al individuo es poco spinozista. ${ }^{13}$ Por el contrario, afirmo que es el propio Spinoza el que caracteriza al individuo de manera intrínseca al identificar la esencia con el esfuerzo por perseverar en el ser que es una expresión de la potencia de la sustancia y que no se justifica por los cuerpos externos. Considero que ambas caracterizaciones responden a la cuestión sobre lo que puede un cuerpo; que consiste en las consideraciones de la digresión física junto con la potencia con la que se esfuerza por perseverar en su ser.

Ahora bien, para poder contestar profundamente a la pregunta por lo que puede un cuerpo debemos considerar tanto las especificidades del conatus como las variaciones de su potencia. Cuando el esfuerzo se refiere a la mente sola se llama "voluntad", cuando se refiere la mente y al cuerpo "apetito" y cuando es apetito con conciencia se llama "deseo" (E III, prop. 9, esc.). Así, "el deseo es el apetito acompañado de la conciencia del mismo" (E III, prop. 9, esc.). Si bien se denomina "voluntad" cuando el conatus se refiere solo a la mente, lo importante es que el conatus es el esfuerzo tanto del cuerpo como de la mente. Para el caso de los individuos humanos, Spinoza sostiene:

12 Quien acentúa la relevancia de los cuerpos exteriores presionando en la conformación de los individuos es Macherey (Cf. Macherey 2014, 218).

13 Así lo sostiene: "The demand that there be something 'inside' the thing, as Barbone puts it, that 'gives unity and unicity to an individual' is, as I see it, deeply unspinozistic. Spinoza's view that the 'form' or 'nature' of an individual consists in its structural coherence (ratio of motion and rest) is presented as an alternative to substantival accounts of individuation. Unlike the latter, Spinoza's account does not depend on the existence of some further feature beyond structural coherence that would set an individual apart from the rest of nature. What makes an individual an individual is just the coherence itself. As Alexandre Matheron put it in his review of Den Uyl's". (Steinberg 2019, 134-135). 
El deseo es la misma esencia o naturaleza de cada uno, en cuanto que se concibe determinada a hacer algo por cualquier constitución suya dada (ver 3/9e). Luego, tal como cada uno es afectado por las causas externas con esta o aquella especie de alegría, tristeza, amor, odio, etc.; esto es, tal como su naturaleza está constituida de uno u otro modo, también es necesario que su deseo sea uno u otro, y que la naturaleza de un deseo difiera de la del otro tanto cuanto difieren entre sí los afectos de los que surge cada uno de esos deseos. (E III, prop. 56, dem.).

Siendo el deseo para el caso de los humanos la esencia misma de cada cual, en cuanto se la concibe determinada a obrar algo en virtud de una constitución cualquiera dada, este afirma solo aquello que la mente es y puede, y no afirma lo que la mente no es o lo que ella no puede (E III, prop. 54 , dem.). El deseo no es la voluntad (en el sentido usual de la palabra) de algo, sino el aspecto actual de la esencia acompañado de conciencia. Ahora bien, para comprender las variaciones de la potencia con la que un individuo ejerce su esfuerzo, debemos conocer el origen y naturaleza de los afectos.

Los afectos son aumentos y disminuciones de la potencia con la que se ejerce el conatus. La primera definición de la tercera parte es una distinción que, en primer lugar, define qué es ser causa adecuada. Afirma Spinoza que causa adecuada es aquella cuyo efecto puede percibirse clara y distintamente por ella misma. Contrariamente, una causa es inadecuada si el efecto no puede entenderse por ella sola (E III, def. 1). En la definición siguiente Spinoza distingue actuar y padecer: actuamos cuando ocurre algo en nosotrxs o fuera de nosotrxs de lo cual somos causa adecuada. Padecemos, en cambio, cuando somos causa parcial (E III, def. 2). Las variaciones de la potencia de un individuo (que es un cuerpo complejo en el atributo extensión y una mente compleja en el atributo pensamiento) aparecen en la definición siguiente que tiene por objeto los afectos: ${ }^{14}$ "por afectos entiendo las afecciones del cuerpo, con las que se aumenta o disminuye, ayuda o estorba, la potencia de actuar de ese mismo cuerpo, y al mismo tiempo las ideas de esas afecciones." (E III, def. 3). Lo que aumenta la potencia corresponde tanto al cuerpo como a la mente. Lo mismo sucede con

$14 \mathrm{Al}$ respecto de la relación entre ideas y las nociones de obrar y padecer sin que jueguen, aún, un rol los afectos afirma María Jimena Solé: "Es claro a partir de esto que las ideas adecuadas son acciones de la mente, mientras que las inadecuadas implican que esta padece. Así lo establece la primera proposición de E III: 'Nuestra alma obra ciertas cosas, pero padece ciertas otras; a saber: en cuanto que tiene ideas adecuadas, entonces obra necesariamente ciertas cosas, y en cuanto que tiene ideas inadecuadas, entonces padece necesariamente ciertas otras' (E III, 1). La lectura que quiero defender aquí se basa en una comprensión rigurosa de esta proposición. Según mi interpretación, Spinoza establece aquí que formar una idea adecuada es actuar, mientras que percibir una idea inadecuada es padecer. Lo que considero fundamental es que esta identificación entre las diferentes clases de ideas y las nociones de obrar y padecer se da, en este primer momento, sin la aparición del concepto de afecto - lo cual ha sido pasado por alto por muchos intérpretes, incluso entre los que enfatizan el carácter práctico de la filosofía spinoziana". (Solé 2019, 34). 
las pasiones tristes: son disminuciones de la potencia tanto del cuerpo como de la mente. Si somos causa adecuada el afecto es una acción; si no, es una pasión.

Lo que puede un individuo, es decir, un cuerpo compuesto finito considerado desde el atributo extensión, no es solo lo que dicta la digresión física, sino también la potencia con la que ejerce su conatus. Potencia que varía según los afectos. Todos los afectos por los que varía la potencia del conatus de un individuo, o, lo que es lo mismo en el atributo extensión, un cuerpo complejo, se derivan de tres primarios: la alegría, la tristeza y el deseo. La alegría es el aumento de la potencia con la que se esfuerza en perseverar en su ser. La tristeza, en cambio, es la disminución de la potencia con la que se esfuerza en perseverar en su ser (E III, prop. 11, esc.). El deseo ya fue definido como el apetito con conciencia, cuya potencia va variando según las alegrías y las tristezas. De estos tres afectos, se desprenden todos los restantes: amor, odio, esperanza, miedo, etc. En cuanto actuamos, solo podemos tener alegría y deseo (E III, prop. 58) y sus derivados. Ningún afecto de tristeza puede referir al actuar (E III, prop. 59), porque en la medida en que una cosa se entristece disminuye su potencia de actuar (E III, prop. 59, dem). Tenemos, de este modo, tres pasiones primarias y solo dos acciones de la misma característica, alegría y deseo, todas las cuales explican las variaciones de la potencia del conatus; es decir, explican cómo varía la potencia del segundo aspecto de la noción de individuo. Potencia que consiste, junto con las consideraciones de la digresión física, en lo que puede un cuerpo.

Es importante aquí no perder de vista la doble atribución (la correlación entre los atributos pensamiento y extensión) al abordar la noción de individuo, el conatus y las variaciones de la potencia. Hay un solo y mismo orden para las afecciones de un cuerpo y las ideas de esas afecciones, un solo y mismo orden para el atributo pensamiento y para el atributo extensión (E II, prop. 7, corol.). Cuando la mente padece el cuerpo tiene una afección pasiva, y cuando la mente actúa el cuerpo también actúa. Así, la potencia con la que un individuo ejerce el esfuerzo por perseverar en el ser es variable, varía según padecimientos y acciones e implica necesariamente tanto al cuerpo como a la mente. En definitiva, la idea de todo cuanto aumenta o disminuye la potencia de actuar de nuestro cuerpo, a su vez, aumenta o disminuye la potencia de actuar de la mente (E III, prop. 11). Habiendo un solo y único orden, la potencia con la que se ejerce la perseverancia en el ser es la misma, ya sea considerada bajo un atributo o el otro. No puede haber una consideración del cuerpo separado de la mente. 
Lo crucial es recordar que tanto la definición de la digresión física como el conatus como las variaciones de la potencia de este implican la doble atribución. Dicho de otra manera: todos los aspectos del individuo implican siempre tanto la mente como el cuerpo. Por todo lo anterior, es imposible que "lo que pueda un cuerpo" esté separado de lo que pueda la mente que es idea de ese cuerpo. Veamos qué afirma explícitamente Spinoza al respecto.

\section{ESCOLIO A LA PROPOSICIÓN 2 DE E III: HISTORIA DE LA INVERSIÓN DE UN ERROR}

En este apartado dedicaré el análisis al escolio a la segunda proposición de la tercera parte; lugar de donde surgieron los malentendidos expresados en el título del presente trabajo: ¿Por qué es inadecuada la frase "nadie sabe lo que puede un cuerpo"? El objetivo es dilucidar dicho escolio contra aquellxs que interpretan la frase "nadie sabe lo que puede un cuerpo" como la imposibilidad de conocimiento sobre los cuerpos, o la potencia ilimitada de ellos.

La contracara de la doble atribución con un solo y mismo orden es que "ni el cuerpo puede determinar al alma a pensar, ni el alma puede determinar al cuerpo al movimiento ni al reposo, ni a alguna otra cosa (si es que la hay)" (E III, prop. 2). Es decir, no puede haber una relación de causalidad entre ambos atributos, no puede haber ninguna interacción entre ellos. Es importante tener en cuenta esta proposición para no perder de vista la doble atribución de las dos caracterizaciones de la noción de individuo mencionadas: como relación y como conatus. La mente y el cuerpo son una sola y misma cosa que se concibe o bien por un atributo o bien por otro. Hay, entonces, un único orden para los individuos, que genera una igualdad entre el orden de las acciones y pasiones del cuerpo y el orden de las acciones y pasiones de la mente (E III, prop. 2, esc.). Sin embargo, la comprensión de la doble atribución de un mismo orden tiene sus complicaciones. Sobre ellas Spinoza se detiene en el escolio de la proposición en cuestión. El problema es que dicho escolio ha dado lugar a una serie de malentendidos a la hora de comprender la doble atribución de los individuos. Afirma Spinoza en el famoso escolio a la segunda proposición de la tercera parte de la Ética:

Pero, aunque estas cosas son tan obvias que no queda motivo alguno de duda, apenas si puedo creer que, si no las confirmo por la experiencia, puedan los hombres ser inducidos a sopesarlas con ecuanimidad. ¡Tan firmemente persuadidos están de que el cuerpo ora se mueve ora reposa ante una simple indicación del alma, y de que hace muchísimas cosas que tan sólo dependen de la voluntad del alma y del arte de excogitar. (E III, prop. 2, esc.). 
En primer lugar, entonces, el prejuicio que aparece es el establece que la mente actúa y el cuerpo obedece. $Y$, aunque no se pueda dudar si se considera "las cosas" que mente y cuerpo son lo mismo desde distintos atributos, sin embargo, el análisis suele ser abordado abrazando este prejuicio; según el cual la mente actúa y el cuerpo padece. Este es el error contra el que argumenta Spinoza. De él se desprende una consecuencia:

Nadie, en efecto, ha determinado por ahora qué puede el cuerpo, esto es, a nadie hasta ahora le ha enseñado la experiencia qué puede hacer el cuerpo por las solas leyes de la naturaleza, considerada como puramente corpórea, y qué no puede a menos que sea determinado por el alma. Pues nadie hasta ahora ha conocido la fábrica del cuerpo con tal precisión que haya podido explicar todas sus funciones. (E III, prop. 2, esc.).

Por un lado, como adelanté, no encontramos en las páginas de la Ética la frase "nadie sabe lo que puede un cuerpo". Lo más parecido es "nadie, en efecto, ha determinado por ahora qué puede el cuerpo". Acá hay un circunstancia crucial: "por ahora". Hay una gran diferencia entre "no se puede determinar lo que puede un cuerpo" y "nadie ha determinado por ahora lo que puede un cuerpo". Si bien por ahora no se ha determinado qué puede hacer el cuerpo en virtud de las solas leyes de su naturaleza; hay que evaluar bien el alcance de este "por ahora". De hecho, a esta altura de la tercera parte, ya contamos con la digresión física de la segunda parte de la Ética, donde Spinoza efectivamente sí explica los cuerpos mediante axiomas, lemas y postulados que corresponden únicamente a su atributo. Con lo cual, con el "por ahora" Spinoza se refiere a otras investigaciones, y no incluye su propia filosofía. Si bien en este pasaje Spinoza se refiere a "lo que ha enseñado la experiencia", la conclusión es que tanto la experiencia como la razón enseñan lo mismo: la no interacción mente-cuerpo, que no es el caso de que la mente mande y el cuerpo obedezca. Continúa un poco más abajo en el escolio:

Además, nadie sabe de qué forma o con qué medios mueve la mente al cuerpo, ni cuántos grados de movimiento puede imprimirle y con qué rapidez puede moverlo. De donde se sigue que, cuando los hombres dicen que esta o aquella acción del cuerpo procede del alma que tiene dominio sobre el cuerpo, no saben lo que dicen y no hacen sino confesar con especiosas palabras que ignoran la verdadera causa de aquella acción. (E III, prop. 2, esc.).

El comienzo de esta cita puede ser confuso. Que nadie sepa la manera en que la mente mueve al cuerpo, ni cuantos grados de rapidez pueda imprimirle no descarta que eso pueda suceder. Sin embargo, la propuesta spinoziana es muy distinta. La afirmación es una argumentación por el absurdo: si la mente moviese al cuerpo, los defensores de este prejuicio sabrían cómo y cuánto movimiento podría imprimirle. Pero no lo saben. En 
la digresión física, en cambio, sí encontramos un lema sobre la interacción entre los cuerpos respecto de "imprimir movimiento", cuando sostiene que un cuerpo ha debido ser determinado al movimiento o al reposo por otro cuerpo, con el que ocurre lo mismo y así hasta el infinito (E II, prop. 13, lem. 3.). A la luz de la tercera parte, este lema cobra mayor relevancia. No es que un cuerpo ha podido ser determinado al movimiento o al reposo por otro cuerpo, sino que ha debido necesariamente ser determinado por otro cuerpo. Lo que se descarta es que haya podido ser determinado al movimiento o al reposo por algo que no sea un cuerpo, por ejemplo, una mente. Aunque el lema 3 no aparezca explícitamente en la demostración de E III, prop. 2, esta proposición es la contracara de dicho lema: la mente no puede determinar al cuerpo al movimiento o al reposo.

De este modo, es evidente por qué nadie sabe cómo la mente mueve al cuerpo ni cuanto movimiento puede imprimirle. Esto no se puede determinar porque no existe tal cosa. Este tipo de problemas, inundados de prejuicios, es lo que ha hecho que por ahora nadie haya determinado lo que puede un cuerpo en virtud de las solas leyes de su naturaleza.

Ahora bien, la idea de que la mente mueve al cuerpo tiene tantxs adeptxs que Spinoza postula algunas posibles objeciones a su postura:

Pero dirán que, sepan o ignoren con qué medios la mente mueve al cuerpo, ellos experimentan en todo caso que, si el alma no fuera apta para reflexionar, el cuerpo sería inerte [...] les pregunto: ¿no enseña también la experiencia que, por el contrario, mientras el cuerpo está inerte, también el alma es inepta para reflexionar? (E III, prop. 2, esc.).

Frente a esta posible objeción, Spinoza responde recurriendo a la mismidad de mente y cuerpo, es decir, a que mente y cuerpo son una sola y misma cosa pero vista desde dos atributos diferentes. Con la respuesta que da a la objeción no solo la refuta, sino que evidencia su postura que niega la existencia de la mente y del cuerpo como dos cosas distintas y separadas, de las cuales la segunda obedece a la primera.

Más adelante, en el mismo escolio, respecto a otra hipotética objeción, Spinoza sostiene: "yo he mostrado ya que ellos no saben qué pueda el cuerpo ni qué se pueda deducir de la sola contemplación de su naturaleza" (E III, prop. 2, esc.). Esto entra en relación con el "por ahora" del comienzo. Habiendo hecho su punto, Spinoza ya no sostiene que por ahora nadie ha determinado lo que puede el cuerpo, sino que ellos ignoran lo que puede el cuerpo. Ellos son quienes sostienen esta causalidad de la mente sobre el cuerpo, y que, por lo tanto, no saben lo que puede un cuerpo en virtud de las solas leyes de su naturaleza. Para Spinoza, en cambio, solo existe una sustancia, un único orden que se expresa en infinitos atributos de los que 
conocemos el pensamiento y la extensión. Para Spinoza mente y cuerpo son una sola y misma cosa, y uno no puede determinar al otro. Más aún, nuestro filósofo establece una serie de axiomas, lemas, postulados, etc., que explican los cuerpos según leyes corpóreas, tal como enumeramos al abordar la digresión física. Lo que puede un cuerpo consta tanto de lo que encontramos en dicha digresión física, como de la potencia con la que algo se esfuerza por perseverar en el ser. En efecto: Spinoza nunca afirma que él no sabe lo que puede un cuerpo, sino que ellos no lo saben. Continúa el escolio:

De suerte que la misma experiencia enseña, no menos claramente que la razón, que los hombres se creen libres por el único motivo de que son conscientes de sus acciones e ignorantes de las causas por las que son determinados; y que, además, las decisiones del alma no son otra cosa que los mismos apetitos, y por eso son tan variadas como la disposición del cuerpo [...]. Todas estas cosas muestran sin duda con claridad que tanto la decisión del alma como el apetito y la determinación del cuerpo son simultáneos por naturaleza o más bien una y la misma cosa, a la que llamamos decisión, cuando es considerada bajo el atributo del pensamiento y explicada por él, y que llamamos determinación, cuando es considerada bajo el atributo de la extensión y deducida de las leyes del movimiento y el reposo. (E III, prop. 2, esc.).

El problema del inicio del escolio reaparece. Hay quienes piensan que la mente decide y el cuerpo obedece porque creen en la causalidad final y en la libertad indeterminada. Para quienes no damos asentimiento a estos prejuicios, la decisión de la mente y la determinación del cuerpo son una sola y misma cosa. La decisión no es una decisión de la mente sobre el cuerpo. Aquí Spinoza cambia el sujeto de enunciación: ya no es "ellos", los que ignoran lo que puede un cuerpo; sino "nosotrxs", que sabemos que mente y cuerpo son una sola y misma cosa. Y entonces llamamos "decisión" o “determinación” según qué atributo la consideremos.

Lo que podemos ver en el escolio es que Spinoza argumenta contra quienes defendían la interacción de la mente y el cuerpo, interacción en el que la primera actúa y manda y el segundo padece y obedece. ${ }^{15}$ Este es el error que denuncia. Spinoza refuta esta postura afirmando la mismidad mente-cuerpo, en tanto una sola y misma cosa según distintos atributos de la única sustancia. Lejos está de sostener aquí nuestro filósofo la indeterminación del cuerpo, o el cuerpo como algo misterioso e ilimitado. Plantear la ilimitación de lo que puede un cuerpo, su potencia absoluta o su independencia de la mente es una inversión del error inicial que partía de un dualismo y jerarquizaba la mente sobre el cuerpo. El error invertido, con

15 Según Maxime Rovere la referencia de Spinoza en esta frase es el Discours sur l'anatomie du cerveau de Steno, quien, de manera socrática, comienza su discurso afirmando que nada sabe sobre el cerebro. De esta manera, Steno rechaza las concepciones previas que había sobre el cerebro (véase Rovere 2017). 
el que Spinoza no discute porque es un error ocasionado en su recepción posterior, también plantea la mente y el cuerpo como cosas distintas, solo que jerarquizando este último. De esta manera, la inversión del error es tan errónea como el error inicial.

Así el famoso "nadie sabe lo que puede un cuerpo" se basa en ideas inadecuadas, como la indeterminación del cuerpo; se basa en la incomprensión de la doble atribución y de la determinación de las cosas. En definitiva, es una afirmación de quienes no comprenden que mente y cuerpo son una sola y misma cosa. Lo que está a la base en esta frase es perder de vista la doble atribución, la no jerarquía entre mente y cuerpo. En primer lugar, las ideas inadecuadas sobre lo que puede un cuerpo, se disipan en la digresión física. Cuestión que, como lo retoma el escolio analizado, no había sido adecuadamente determinada hasta ese momento. Esta es la primera respuesta al problema: lo que puede un cuerpo en virtud de las solas leyes de su naturaleza lo explicó Spinoza en la digresión física; considerando el individuo según su primer aspecto, en tanto relación de movimiento y reposo.

Ahora bien, según el segundo aspecto del individuo, podemos dar una respuesta más completa a la pregunta por lo que puede un cuerpo; considerando el individuo finito como un cuerpo complejo, este cuerpo tiene conatus. Lo que puede un cuerpo no es solo lo que se sigue de la digresión física, lo que puede un cuerpo es algo intrínseco en tanto él es una expresión de la potencia de la sustancia. Esta es la segunda respuesta a la pregunta por lo que puede un cuerpo: lo que puede es su potencia, la potencia con la que ejerce su esfuerzo por perseverar en el ser que varía según los afectos. Una potencia que no es absoluta, que siempre aumenta o disminuye.

El escolio finaliza afirmando que los que creen que hacen cualquier cosa por libre decisión de la mente sueñan con los ojos abiertos. Así también - podemos agregar - sueñan con los ojos abiertos quienes creen hacer algo por libre determinación del cuerpo; o, mejor dicho, por indeterminación del cuerpo.

\section{CONSIDERACIONES FINALES}

Con la frase "nadie, en efecto, ha determinado por ahora qué puede el cuerpo [...] por las solas leyes de la naturaleza, considerada como puramente corpórea, y qué no puede a menos que sea determinado por la mente" Spinoza pretendía refutar a quienes postulaban una dualidad mente-cuerpo con acción de la primera sobre el segundo. Este era el error original. Sin embargo esta oración abrió un nuevo frente: quienes reformulan este 
escolio con la frase "nadie sabe lo que puede un cuerpo", para plantear la indeterminación o ilimitación de los cuerpos. Así, se olvida nuevamente la mismidad de la mente y el cuerpo; invirtiendo el error al plantear la indeterminación del cuerpo, o su potencia absoluta, jerarquizándolo y distinguiéndolo de la mente. De esta manera, ambos frentes (tanto la postura con la que discutía Spinoza como la recepción de este escolio) caen en un dualismo jerarquizante, ya sea con jerarquía de la mente o del cuerpo.

En el medio de toda esta polémica, la noción de individuo se revela como la clave para salir del falso dilema dualista. Un individuo, considerado desde el atributo extensión es un cuerpo complejo. Cuerpo que mantiene una relación de movimiento y reposo de sus partes y que puede ciertas cosas en virtud de las solas leyes de su naturaleza, tal como lo explica la digresión física. Lo mismo vale para el atributo pensamiento. Pero también un individuo es conatus (apetito si lo referimos a la mente y al cuerpo y deseo si es el caso de que haya conciencia de él). Conatus cuya potencia varía según los afectos. Así, el esfuerzo por perseverar en el ser otorga un criterio intrínseco a la individualidad spinoziana, supliendo la insuficiencia de la digresión física. En definitiva tanto la caracterización del individuo como relación como la del individuo como conatus implican ambos atributos, implican el pensamiento y la extensión; sin jerarquía entre ellos. Y ambas caracterizaciones responden a la pregunta por lo que puede un cuerpo sin caer en jerarquías de un atributo sobre el otro.

Ni la mente manda y el cuerpo obedece (o la mente actúa y el cuerpo padece), ni el cuerpo tiene una potencia indeterminada o absoluta. Ambos son una misma cosa (desde distintos atributos) que actúan y padecen al unísono. Así, lo que puede un cuerpo no solo es lo que dictan los axiomas, lemas, definición y postulados de la digresión física. Lo que puede un cuerpo es también su potencia, la potencia con la que ejerce su esfuerzo por perseverar en el ser. Potencia variable de una cosa (cuerpo complejo en el atributo extensión) finita. Por todo lo anterior, la frase "nadie sabe lo que puede un cuerpo" corresponde a ideas inadecuadas de la imaginación, ideas que arrastran innumerables prejuicios y que pierden un eje central de la filosofía spinoziana: la mismidad mente-cuerpo y la no jerarquía entre ellos. 


\section{BIBLIOGRAFIA}

Aguilar, Claudia. 2019. Resignificación, resistencia y alianzas. Una propuesta desde la teoría de la individualidad spinoziana. Buenos Aires: RAGIF ediciones

Deleuze, Gilles. 1999. Spinoza y el problema de la expresión. Barcelona: Muchnik. 2003. En medio de Spinoza. Buenos Aires: Cactus.

Gueroult, Martial. 1974. Spinoza. L'âme (Éthique, 2). Paris: Aubier-Montagne. Jabase, Leila. 2020. "Individuación y conatus: puntos de intersección entre Spinoza y Leibniz". In Felipe Jardim (ed.), Spinoza Filosofía \& Liberdade . Río de Janeiro: Editora PUC Rio. En prensa.

Leibniz, Gottfried Wilhelm, Sämtliche Schriften und Briefe, DarmstadtLeipzig-Berlin: Akademie Verlag, 1923-sig.

Lin, Martin. 2005. "Memory and personal Identity in Spinoza". Canadian Journal of Philosophy 35-2: 243-268.

Macherey, Pierre. 2014. Hegel o Spinoza. Buenos Aires: Tinta Limón.

Peterman, Alison. 2007. “The 'Physical' Interlude”. In Yitzhak Melamed (ed.): Spinoza's Ethics. A Critical Guide. Cambridge: Cambridge University Press: 102-120.

Rovere, Maxime. 2017. Le Clan Spinoza. Paris: Flammarion.

Solé, María Jimena. 2019. "El conocimiento como acción. Exploración del concepto de filosofía en Spinoza”. Síntesis. Revista de filosofía 2-1: 2344.

Spinoza, Baruch. 2019. Ética demostrada según el orden geométrico (Atilano Domínguez trad.). Madrid: Guillermo Escolar.

Steinberg, Justin. 2019. "Bodies Politic and Civic Agreement". In Aurelia Armstrong, Keith Green \& Andrea Sangiacomo (eds.), Spinoza and Relational Autonomy. Being with Others. Edinburgo: Edinburgh University Press: 132-148.

Williams, Caroline. 2019. "Revisiting Spinoza's Concept of Conatus: Degrees of Autonomy". In Aurelia Armstrong, Keith Green y Andrea Sangiacomo (eds.), Spinoza and Relational Autonomy. Being with Others. Edinburgo: Edinburgh University Press: 115-131. 